\title{
Planck constraints on secret neutrino interactions
}

\section{Francesco Forastieri*}

Dipartimento di Fisica e Scienze della Terra, Università degli Studi di Ferrara, via Giuseppe Saragat 1, I-44122 Ferrara, Italy

Istituto Nazionale di Fisica Nucleare, Sezione di Ferrara, via Giuseppe Saragat 1, I-44122

Ferrara, Italy

E-mail: frsfnc2@unife.it

\begin{abstract}
We present a preliminary study oriented to evaluate the sensitivity of the latest Planck 2015 data release to non-standard self interacts of neutrinos. We consider the case of massless neutrinos whose interaction is mediated by a massless pseudoscalar boson. In this scenario neutrinos decouple form the rest of the primordial fluid as described in standard cosmology, but later, depending on the strength of the interaction, recouple and start to scatter among themselves. This behaviour leaves an imprint on the angular power spectra (APS) of the cosmic microwave background (CMB), moreover the 2015 Planck release includes the high- $\ell$ polarization data which can improve the constraint on the coupling constant $g_{\mathrm{rm}}$ of the secret interaction. The possible improvement in constraining the strength of the new interaction seems to be important and worthy of being thorough.
\end{abstract}

Neutrino Oscillation Workshop

4 - 11 September, 2016

Otranto (Lecce, Italy)

\footnotetext{
*Speaker.
} 


\section{Introduction}

Neutrinos are included in the Standard cosmological model $(\Lambda \mathrm{CDM})$ as part of the relativistic energy content along with photons. This component dominates and drives the expansion of the universe until the radiation-matter energy density ratio $\left(\rho_{\gamma} / \rho_{\text {matter }}\right)$ is larger than one. Laboratory experiments show that neutrinos undergo only weak interactions, thus, due to the expansion, they decouple form the rest of the primordial fluid at $T \simeq 1 \mathrm{MeV}$, when scattering processes due to weak interactions go out of equilibrium. Neutrino decoupling can be thought as an instantaneous process, this translates into a thermal distribution leading to the preservation of a Fermi-Dirac spectrum [1]. From this moment neutrinos are free to stream following the geodesics, this behaviour,, impacting on the dynamics of the fluid perturbations, contributes to wash out perturbations smaller than the free-streaming length. The presence of relativistic extra degrees of freedom $\left(N_{\text {eff }}\right)$ affects the CMB by changing the redshift of radiation-matter equality and by modifying the Silk damping scale, leaving an imprint on the temperature and polarization $\mathrm{CMB}$ anisotropies power spectra [2]. This leads to a strong detection of extra relativistic content widely compatible with three light massive neutrinos. Nowadays cosmological data, in particular the observations of the Cosmic Microwave Background radiation, thanks to Planck as well as ground based experiments, are extremely precise and provide a suitable channel to study some extensions of $\Lambda \mathrm{CDM}$ and of the standard model of particles (SM). In this work we study the effect of a non-standard neutrino self-interaction mediated by a light or massless pseudoscalar boson $\phi$. This interaction might be related to the Majorana nature of neutrinos. In this picture the neutrino mass generation, the so called "Seesaw" mechanism, is more elegant and natural with respect to the Dirac one [2, 3, 4], it requires to "sacrifice" lepton number conservation which, however, might not be a fundamental quantity.

\section{Pseudoscala interaction impacts on the power spectra}

As said previously, according to the standard model of particles, neutrinos undergo only weak interactions and, in cosmology, they are treated as a collisionless fluid after decoupling, i.e. the contribution due to scattering precesses is negligible. Thus, at times of interest for CMB and structure formation, there is no need to take into account collisional terms in any dynamical process involving neutrinos. However, if we add a secret self-interaction able to increase the viscosity of the neutrino fluid, this assumption is no longer valid. Introducing a pseudoscalar interaction among neutrinos described by the Lagrangian $[5,6,7]$ :

$$
\mathscr{L}=h_{i j} \bar{v}_{i} v_{j} \phi+g_{i j} \bar{v}_{i} \gamma_{5} v_{j} \phi+h . c .,
$$

we are going to consider neutrino-neutrino scattering mediated by a massless scalar boson $(\phi)$ exchange $(v+v \leftrightarrow v+v)$. The scattering cross section is the standard one for a binary process mediated by a massless boson [8]:

$$
\sigma_{\text {bin }} \sim g^{4} / s,
$$

where $\sqrt{s}$ is the center of mass energy and $g$ is the largest entry of the Yukawa matrix. This cross section is valid only in the ultrarelativistic limit which is a good approximation for the purpose of this study. Thus, in thermal equilibrium, the rate for a binary process is:

$$
\Gamma_{\text {bin }}=\left\langle\sigma_{\text {bin }} v\right\rangle n_{\text {eq }} \propto g^{4} T,
$$


where the neutrino abundance at the equilibrium is proportional to $T^{3}$ and we have used $\sqrt{s} \sim T$. Comparing this scattering rate with the Hubble rate shows that the $\Gamma_{\text {bin }} / H$ ratio increases during the universe expansion, this suggests to a neutrino-neutrino recoupling at late times with respect to standard neutrino decoupling. In order to study the impact of this interaction on the anisotropies power spectrum (APS) we used the public code [9]. We modified the neutrino fluid equations introducing a scattering term in the right-hand side of the Boltzmann equation:

$$
\frac{\partial \Psi}{\partial \tau}+i \frac{q(\vec{k} \cdot \hat{n})}{\varepsilon} \Psi+\frac{d \ln f_{0}}{d \ln q}\left[\dot{\phi}-i \frac{q(\vec{k} \cdot \hat{n})}{\varepsilon} \Psi\right]=\frac{1}{f_{0}} \hat{C}[f],
$$

where $\tau$ is the conformal time, $\hat{C}[f] \simeq \Gamma \delta f$ and $\Gamma=a g_{\text {eff }}^{4} T$ is the effective scattering rate. Considering the infinite hierarchy which arises form the harmonic expansion (see [10] for details), the interaction does not affect the first two orders: monopole $(\ell=0)$ due to particle number conservations, dipole $(\ell=1)$ due to momentum conservation. However, from the anisotropic shear $(\ell=2)$ onwards, the scattering term reduces, within the limits of tight coupling, the higher momenta [11]. This imprint propagates to lower momenta and affects the density perturbations $(\ell=0)$ basically increasing their power. The visible effect on the APS is a magnification over all the multipoles, it is clearly visible in Fig.1, both for TT and TE spectra. We choose to investigate two different values for the coupling constant: $g_{\text {eff }}=1.78 \times 10^{-7}$ (red dashed line) and $g_{\text {eff }}=2.1 \times 10^{-7}$ (red dotted line). We choose these values for the strength of the interaction in light of the most recent constraints present in literature $[12,13,14,15]$. For both cases the residuals with respect to the $\Lambda C D M$ model are larger than the cosmic variance and the Planck 2015 uncertainties up to $\ell \sim 2100$. This appears to be a good hint regarding the sensitivity of the cosmic microwave background temperature and polarization APS to the presence of the secret interaction. In particular Planck 2015 uncertainties in the TE power spectrum are good enough to allow a considerable improvement constraining the strength of the secret interaction.

\section{Conclusions}

We investigated a secret pseudoscalar interaction between neutrinos that can leave important signatures on the cosmic microwave background APS. This interaction reduces the shear of the neutrino fluid and consequently increases the density and pressure perturbations. The effects are visible over a wide range of multipoles of the CMB APS and in the selected cases are larger than the experimental uncertainties. We have compared it with the most recent public data of the Planck collaboration, putting our attention on the improvements of the Planck 2015 release [16] with respect the 2013 one [17]. This increase the interest on possible extensions of the standard cosmological model and of the standard model of particles that can be constrained by cosmological data and suggests a deeper Monte Carlo based analysis. 

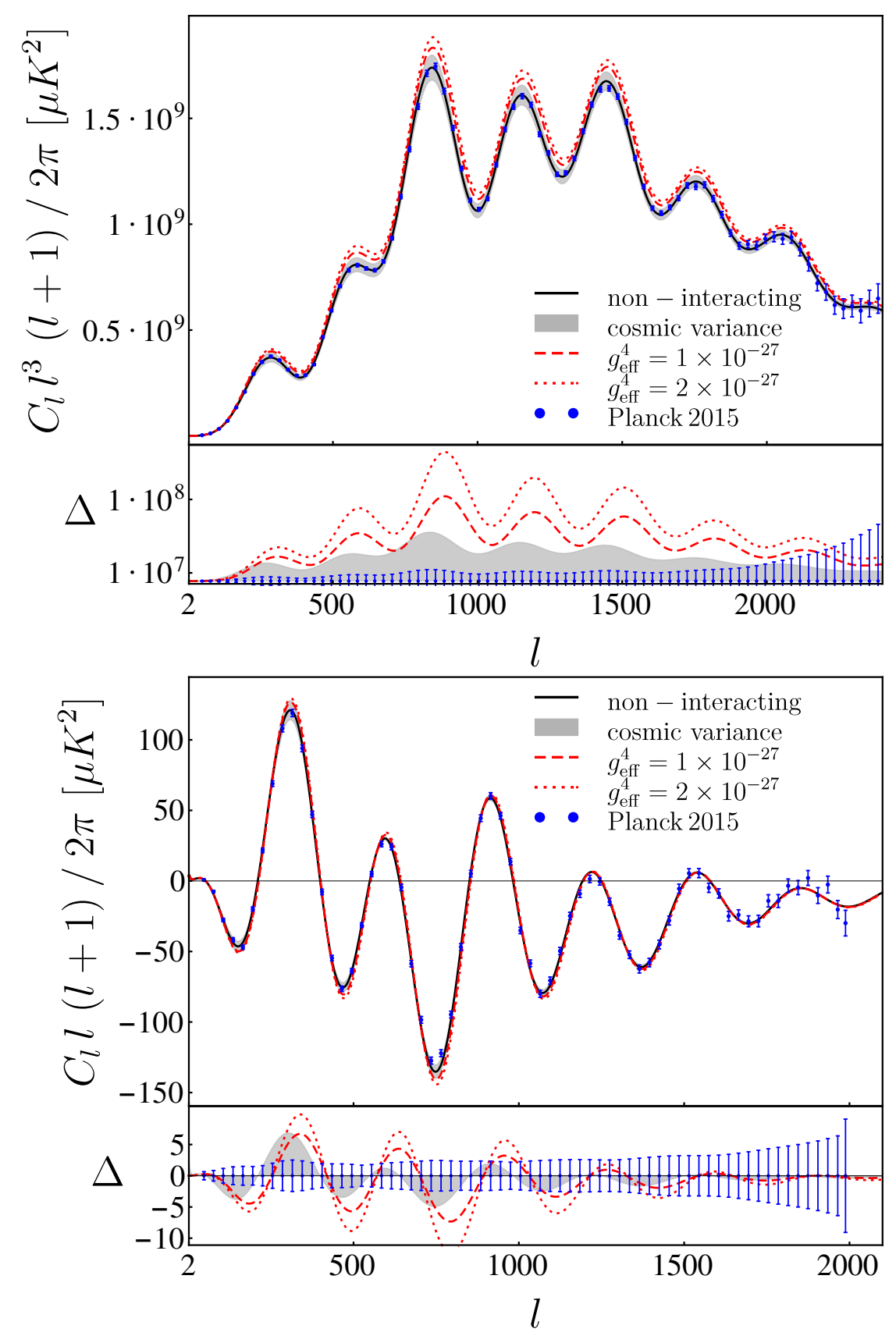

Figure 1: In the upper panel we show the TT power spectrum and in the lower panel the TE power spectrum. In each figure we show the APS for two different models: the standard $\Lambda$ CDM (black solid line) and $\Lambda$ CDM $+g_{\text {eff }}^{4}$ (red line), in this last model we perform two different cases using $g_{\text {eff }}^{4}=1 \times 10^{-27}$ (red dashed line) and $g_{\text {eff }}^{4}=2 \times 10^{-27}$ (red dotted line). The gray shaded zone is the associated cosmic variance and blue dots represent the Planck 2015 best-fit data [16] with the corresponding uncertainties. In the lower panel we present the residuals among the interacting cases and the standard one plotted against the cosmic variance and the Planck uncertainties.

\section{References}

[1] A. Cuoco, J. Lesgourgues, G. Mangano and S. Pastor, Phys. Rev. D 71, 123501 (2005) [astro-ph/0502465]. 
[2] J. Lesgourgues, et al. "Neutrino cosmology." Cambridge University Press, 2013.

[3] J. Lesgourgues and S. Pastor, Adv. High Energy Phys. 2012, 608515 (2012) [arXiv:1212.6154 [hep-ph]].

[4] J. Lesgourgues and S. Pastor, Phys. Rept. 429, 307 (2006) [astro-ph/0603494].

[5] Y. Chikashige, R. N. Mohapatra and R. D. Peccei, Phys. Lett. B 98, 265 (1981).

[6] J. Schechter and J. W. F. Valle, Phys. Rev. D 25, 774 (1982).

[7] G. B. Gelmini and M. Roncadelli, Phys. Lett. B 99, 411 (1981).

[8] Peskin, Michael, and Dan Schroeder. "An introduction to quantum field theory." 1995.

[9] A. Lewis, A. Challinor and A. Lasenby, Astrophys. J. 538, 473 (2000).

[10] C. P. Ma and E. Bertschinger, Astrophys. J. 455 (1995) 7 [astro-ph/9506072].

[11] F. Forastieri, M. Lattanzi and P. Natoli, JCAP 1507, no. 07, 014 (2015) doi:10.1088/1475-7516/2015/07/014 [arXiv:1504.04999 [astro-ph.CO]].

[12] N. F. Bell, E. Pierpaoli and K. Sigurdson, Phys. Rev. D 73, 063523 (2006) [astro-ph/0511410].

[13] A. Basboll and S. Hannestad, JCAP 0701, 003 (2007) [hep-ph/0609025].

[14] F. Y. Cyr-Racine and K. Sigurdson, Phys. Rev. D 90, no. 12, 123533 (2014) [arXiv:1306.1536 [astro-ph.CO]].

[15] M. Archidiacono and S. Hannestad, JCAP 1407, 046 (2014) [arXiv:1311.3873 [astro-ph.CO]].

[16] N. Aghanim et al. [Planck Collaboration], Astron. Astrophys. 594 (2016) A11 doi:10.1051/0004-6361/201526926 [arXiv:1507.02704 [astro-ph.CO]].

[17] P. A. R. Ade et al. [Planck Collaboration], Astron. Astrophys. 571 (2014) A15 doi:10.1051/0004-6361/201321573 [arXiv:1303.5075 [astro-ph.CO]]. 